\title{
Ethylene Signaling Is Important for Isoflavonoid-Mediated Resistance to Rhizoctonia solani in Roots of Medicago truncatula
}

\author{
Yao Liu, ${ }^{1,2}$ Samira Hassan, ${ }^{3}$ Brendan N. Kidd, ${ }^{1}$ Gagan Garg, ${ }^{1}$ Ulrike Mathesius,,${ }^{3,+}$ Karam B. Singh,,${ }^{1,4}$ and \\ Jonathan P. Anderson ${ }^{1,4,+}$ \\ ${ }^{1} \mathrm{CSIRO}$ Agriculture and Food, Floreat, Western Australia; ${ }^{2}$ Rice Research Institute, Sichuan Agricultural University, Chengdu, \\ China; ${ }^{3}$ Research School of Biology, Australian National University, Canberra, Australian Capital Territory; and ${ }^{4}$ The UWA \\ Institute of Agriculture, University of Western Australia, Crawley, Western Australia
}

Accepted 12 May 2017.

\begin{abstract}
The root-infecting necrotrophic fungal pathogen Rhizoctoniasolani causes significant disease to all the world's major food crops. As a model for pathogenesis of legumes, we have examined the interaction of $R$. solani AG8 with Medicago truncatula. RNAseq analysis of the moderately resistant $M$. truncatula accession A17 and highly susceptible sickle (skl) mutant (defective in ethylene sensing) identified major early transcriptional reprogramming in A17. Responses specific to A17 included components of ethylene signaling, reactive oxygen species metabolism, and consistent upregulation of the isoflavonoid biosynthesis pathway. Mass spectrometry revealed accumulation of the isoflavonoidrelated compounds liquiritigenin, formononetin, medicarpin, and biochanin A in A17. Overexpression of an isoflavone synthase in $M$. truncatula roots increased isoflavonoid accumulation and resistance to $R$. solani. Addition of exogenous medicarpin suggested this phytoalexin may be one of several isoflavonoids required to contribute to resistance to $R$. solani. Together, these results provide evidence for the role of ethylene-mediated accumulation of isoflavonoids during defense against root pathogens in legumes. The involvement of ethylene signaling and isoflavonoids in the regulation of both symbiont-legume and pathogen-legume interactions in the same tissue may suggest tight regulation of these responses are required in the root tissue.
\end{abstract}

Rhizoctonia solani is a root-infecting pathogen that causes substantial disease impacts to a broad array of the world's most important crops, including rice, maize, wheat, barley, soybean, and alfalfa (Hane et al. 2014). Isolates are classified into anastomosis groups (AG) based on vegetative compatibility and range from having a specific to a very broad host range. Conventional breeding has had limited efficacy for introducing genetic resistance in any of the crops in which $R$. solani causes disease. Furthermore, the broad host range of some isolates

Sequence data can be found in the Gene Expression Omnibus database under accession number GSE94260 and the ArrayExpress database under accession number E-MTAB-5473.

${ }^{\dagger}$ Corresponding authors: J. Anderson; E-mail: Jonathan.Anderson@csiro.au and U. Mathesius; E-mail: Ulrike.Mathesius@anu.edu.au

*The $e$-Xtra logo stands for "electronic extra" and indicates that eight supplementary figures and four supplementary tables are published online.

(C) 2017 The American Phytopathological Society limits the utility of cultural controls such as crop rotation. Despite the significant damage caused by the $R$. solani species complex on the world's food supply, comparatively little is known about the components of an effective plant defense response or the pathogenicity mechanisms the fungus employs to cause disease. An understanding of these defense mechanisms may guide novel strategies to enhance resistance in crop plants.

Legumes make up a substantial component of dietary protein for humans and are a staple food source for many people in developing countries (Graham and Vance 2003). Legume crops have consistently suffered high losses to disease (Tivoli et al. 2006), with some of the worlds' largest legume crops, such as soybean and chickpea, facing disease pressure from $R$. solani. For example, a strain of $R$. solani AG1-IA that causes Rhizoctonia foliar blight reportedly caused a loss of 104,700 metric tons of soybean in the United States, India, and Brazil in 1994 (Wrather et al. 1997).

The model legume Medicago truncatula has been adopted as a model species for many plant-microbe and plant-insect interaction studies, including with Aphanomyces euteiches (Bonhomme et al. 2014; Djébali et al. 2013), Fusarium oxysporum (Thatcher et al. 2016), Erysiphe pisi (Foster-Hartnett et al. 2007), $R$. solani AG8 and AG6 isolates (Anderson and Singh 2011; Anderson et al. 2013), Ralstonia solanacearum (Vailleau et al. 2007), blue green aphid, spotted alfalfa aphid, pea aphid, and cowpea aphid (Gao et al. 2007, 2008; Kamphuis et al. 2012a, 2013; Klingler et al. 2007; Stewart et al. 2009), and the chewing insect Spodoptera littoralis and piercing-sucking insect Tetranychus urticae (Leitner et al. 2005). M. truncatula has also been widely utilized to study symbiotic interactions with bacterial and fungal species such as Sinorhizobium meliloti (Jones et al. 2007; Ng et al. 2015), Glomus versiforme, or Rhizophagus irregularis (Bravo et al. 2016) and several others. A wide array of genetic and genomic resources are available for $M$. truncatula, including a genome sequence, a transformation system, recombinant inbred line populations, and several reverse genetics populations (Cheng et al. 2014; Hoffmann et al. 1997; Young et al. 2011), making it an attractive host for a model pathosystem.

We have previously shown the resistance or susceptibility of $M$. truncatula to different isolates of $R$. solani is conferred by different regions of the host genome. Moderate resistance to an AG6 isolate could be mapped to a quantitative trait locus on linkage group 4/8, however the parental lines did not differ in response to other $R$. solani isolates belonging to AG8 (bare patch and hypocotyl rot) or AG11 (hypocotyl rot) (Anderson 
et al. 2013). Resistance to the cereal-, brassica-, and legumeinfecting isolate AG8 and the legume-specific isolate AG11 was found to be dependent on ethylene signaling (Anderson et al. 2013; Penmetsa et al. 2008). Members of the class IX AP2/ERF family of transcription factors were found to be responsive to $R$. solani AG8 inoculation in the moderately resistant accession A17, which is the reference genotype for M. truncatula (Anderson et al. 2010). In contrast, sickle ( $\mathrm{skl}$ ), an ethylene-insensitive mutant in the A17 background with a point mutation in the homolog of the Arabidopsis EIN2 gene, is highly susceptible to AG8 and showed no transcriptional response of these genes (Anderson et al. 2010; Penmetsa et al. 2008). The Arabidopsis EIN2 gene encodes a central regulator of the ethylene signaling pathway with downstream defense responses activated through the ethylene response factor (ERF) family of transcription factors (Bent et al. 1992; Fujimoto et al. 2000). Overexpression of individual class IX ERF genes in $M$. truncatula roots enhanced resistance to $R$. solani and another root-infecting necrotroph, Phytophthora medicaginis (Anderson et al. 2010). Although ethylene-mediated defenses contribute to moderate resistance to root-infecting necrotrophs and can enhance resistance further when overexpressed, the underlying mechanism of resistance is presently unknown.

In this study, we further elucidated the mechanisms underlying the function of ethylene in the defense of legumes against $R$. solani using in-depth RNA sequencing to compare moderately resistant $M$. truncatula wild type and the highly susceptible skl mutant after infection with $R$. solani AG8. Substantial transcriptional regulation was observed, especially in A17, following R. solani challenge. Among these changes, the

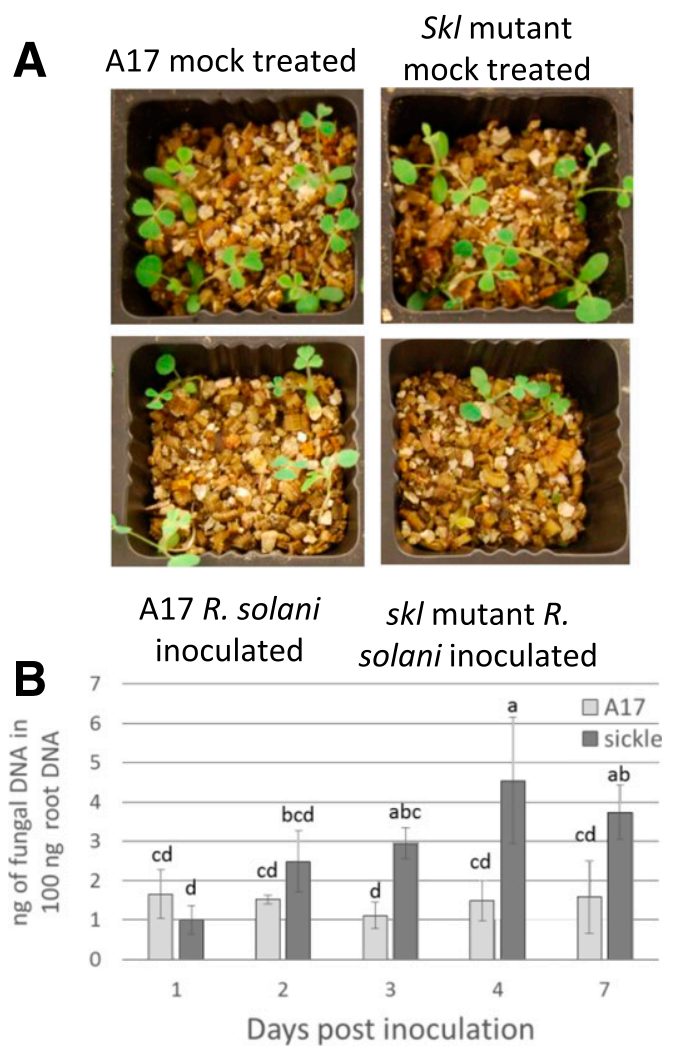

Fig. 1. Performance of $\mathrm{A} 17$ and the $s k l$ mutant after mock treatment or inoculation with Rhizoctonia solani AG8. A, Photographs of pots at 7 days after inoculation or mock treatment. Each was planted with four healthy germinated seedlings at time 0 . B, Quantification of $R$. solani genomic DNA relative to Medicago truncatula genomic DNA in inoculated roots of A17 and the skl mutant. Columns not connected by the same letter are significantly different according to analysis of variance, $P<0.05, n=3$. pathway leading to synthesis of isoflavonoids, including the phytoalexin medicarpin, was specifically up-regulated in A17. To further understand the potential involvement of ethylenemediated accumulation of isoflavonoids in defense responses, metabolite analysis was conducted and the impact of overexpression of ISOFLAVONE SYNTHASE (IFS) and the addition of exogenous medicarpin was analyzed. Together, these experiments demonstrate a connection between ethylene signaling, isoflavonoid synthesis, and resistance to $R$. solani that may be adapted in other crops for enhancing resistance to this destructive pathogen.

\section{RESULTS}

\section{The skl mutant supports higher levels} of $R$. solani growth in planta.

The $s k l$ mutant has been shown to be susceptible to the rootinfecting fungal pathogen Rhizoctonia solani AG8 (Penmetsa et al. 2008). To confirm the moderate resistance of A17, susceptibility of $s k l$, and select informative time points for RNAseq analysis, quantitative polymerase chain reaction (qPCR) was used to measure the relative amount of pathogen DNA in host tissue (Fig. 1). The susceptibility of $s k l$ was first observed by a significantly higher pathogen DNA load in infected $s k l$ compared with A17 at 3 days after $R$. solani infection and this continued through to the last time point at 7 days after inoculation. To gain an insight into early defenses that differ between A17 and skl, the 2-days postinoculation (dpi) time point was chosen for an early stage sample, while the 7-dpi sample was chosen as an advanced stage of infection.

$s k l$ shows changes in basal gene expression relative to A17.

To gain an understanding of the scale and functions of gene expression changes caused by the $s k l$ mutation, global gene expression was compared between A17 and $s k l$ seedlings in the absence of biotic stress. The $s k l$ mutant has several phenotypic differences such as root and hypocotyl elongation (Penmetsa et al. 2008) and this is reflected in a large number of significantly differentially regulated genes in roots of comparable age seedlings (Fig. 2). In total, 233 and 409 genes showed higher expression in $s k l$ at 2- and 7-dpi time points respectively, with 90 of these genes higher in $s k l$ at both time points. In comparison, downregulation of gene expression in $s k l$ was less pronounced, with 120 genes lower at 2 dpi and 245 showing lower expression in skl than A17 at the 7-dpi time point. Despite the large number of genes differentially expressed between A17 and skl, very few gene ontology (GO) terms were significantly overrepresented in either the upregulated or downregulated gene lists in $s k l$. Those genes that were expressed to a lower degree in $s k l$ at the 2-dpi time point showed significantly more association with iron binding and oxidoreductase functions, while those expressed to a higher degree in $s k l$ at the 7-dpi time point showed a significant overrepresentation of the response to chemical stimulus GO term (Supplementary Fig. S1).

\section{Transcriptional reprogramming}

\section{in $R$. solani-infected M. truncatula roots.}

Comparison of gene expression levels between mock-treated and $R$. solani-infected roots using RNAseq revealed major changes in gene expression levels in A17 and a reduced response in $s k l$ that was particularly evident at 7 days after inoculation (Fig. 3). Overall, 501 genes were significantly up-regulated in A17 following $R$. solani challenge, with 364 being specific to the 7-dpi time point (Fig. 3A). The response in $s k l$ was smaller, with only 193 genes significantly upregulated with a small degree of overlap observed between the 2- and 7-day time points. Genes that were down-regulated following $R$. solani inoculation also 
showed a high degree of time point specificity, and a similar magnitude of downregulation was observed in both A17 and $s k l$ at both time points (Fig. 3B).

To understand what these changes in gene expression may relate to, a singular enrichment analysis for overrepresentation of GO categories (Du et al. 2010) was conducted. Genes that were specifically up-regulated in A17 in response to $R$. solani were enriched in GO categories related to reactive oxygen species and responses to biotic stress (Supplementary Fig. S2). However there were no significantly overrepresented GO categories in the genes specifically upregulated in skl. To further dissect the pathways that are activated in A17 and $s k l$ in response to $R$. solani, KOBAS and MapMan were used. MapMan is a tool to overlay previously computed differential gene expression values over graphical representations of cellular pathways to assist in identification of pathways that may be co-ordinately regulated. This approach highlighted differential expression of genes associated with the response to biotic stress (Supplementary Fig. S3). As anticipated, a clear difference in the activation of the ethylene pathway was discernible between A17 and skl at both time points, with A17

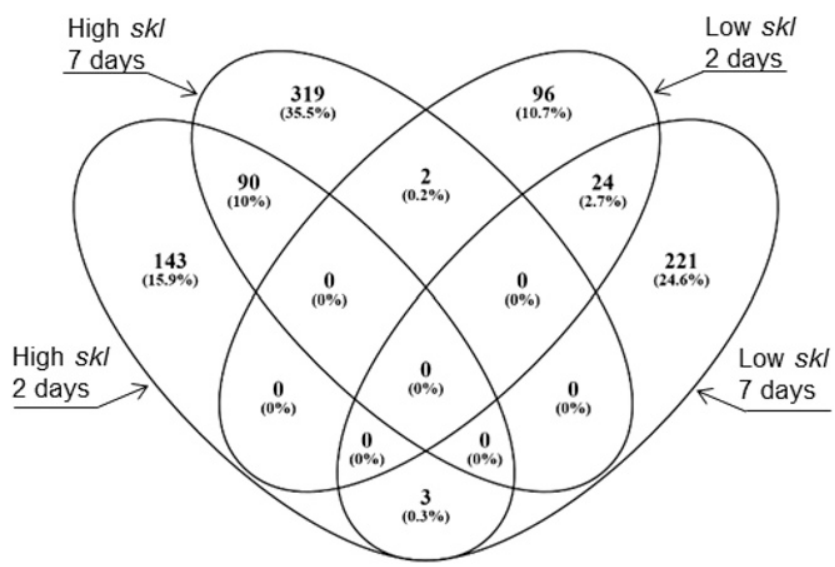

Fig. 2. Differential gene expression between roots of A17 and $s k l$ at the 2and 7-day time points in the absence of the pathogen. Differentially expressed genes have a false discovery rate $<0.05$ and $\log 2$-fold change greater than 1 or less than -1 . The number of differentially expressed genes are shown in each sector. The percentages of all differentially expressed genes are presented in parentheses. High $s k l$ indicates genes with higher expression in $s k l$ than in A17, low $s k l$ indicates genes with lower expression in $s k l$ than in A17. recruiting this pathway early and with greater magnitude by 7 days after infection. Furthermore, although A17 is upregulating a similar number of oxidative stress-related genes (redox state, peroxidases, and glutathione- $S$-transferases) as $s k l$ at $2 \mathrm{dpi}$, A17 has induced many more of these gene categories at 7 dpi compared with $s k l$. Also notable was a difference in the recruitment of secondary metabolism pathways in A17 versus $s k l$. The KOBAS 2.0 webserver takes protein sequences of differentially expressed genes and returns overrepresented Kyoto Encyclopedia of Genes and Genomes (KEGG) pathways associated with those genes relative to a precomputed $M$. truncatula background, using Fisher's exact test with Benjamini and Hochberg false discovery rate (FDR) correction (Xie et al. 2011). Interestingly, KOBAS analysis revealed a specific upregulation of the flavonoid biosynthesis pathway at 2 dpi in A17, whereas $s k l$ had upregulation of nitrogen and lipid metabolism at 2 dpi (Supplementary Table S1). At $7 \mathrm{dpi}$, both A17 and $s k l$ had upregulation of elements of flavonoid and phenlypropanoid biosynthesis.

\section{The expression of flavonoid biosynthesis pathways differs between A17 and $s k l$.}

Numerous genes with flavonoid synthesis related functions were specifically up-regulated in A17. In particular, several genes in the pathway leading to the production of isoflavonoids, such as medicarpin, were specifically induced in A17 and not in skl (Fig. 4A). Moreover, genes encoding enzymes involved in side branches of the pathway, such as that leading to the flavonol kaempferol, were not up-regulated in A17 or in $s k l$. To confirm the gene expression patterns observed from the RNAseq analysis, qPCR was also used to follow expression changes of genes in the isoflavonoid pathway following inoculation of A17 and skl with $R$. solani. Several gene families relating to the production of isoflavonoids had members that showed specific upregulation in A17 following $R$. solani infection at 2- or 7-dpi time points relative to the noninfected mock plants (Fig. 4B and C; Supplementary Fig. S4; Supplementary Table S2). In contrast, a significant reduction in several flavonoid genes relative to A17 at the 2-dpi time point was observed in $s k l$ and, with the exception of a FLAVONE SYNTHASE (FNS) gene, a significant induction of flavonoid genes by $R$. solani infection was not observed in $s k l$ at either time point. The induction of the flavone synthase gene $(F N S)$ in $s k l$ by $R$. solani infection suggests substrates may be shunted into different branches of secondary metabolism than in A17 (Fig. 4C).
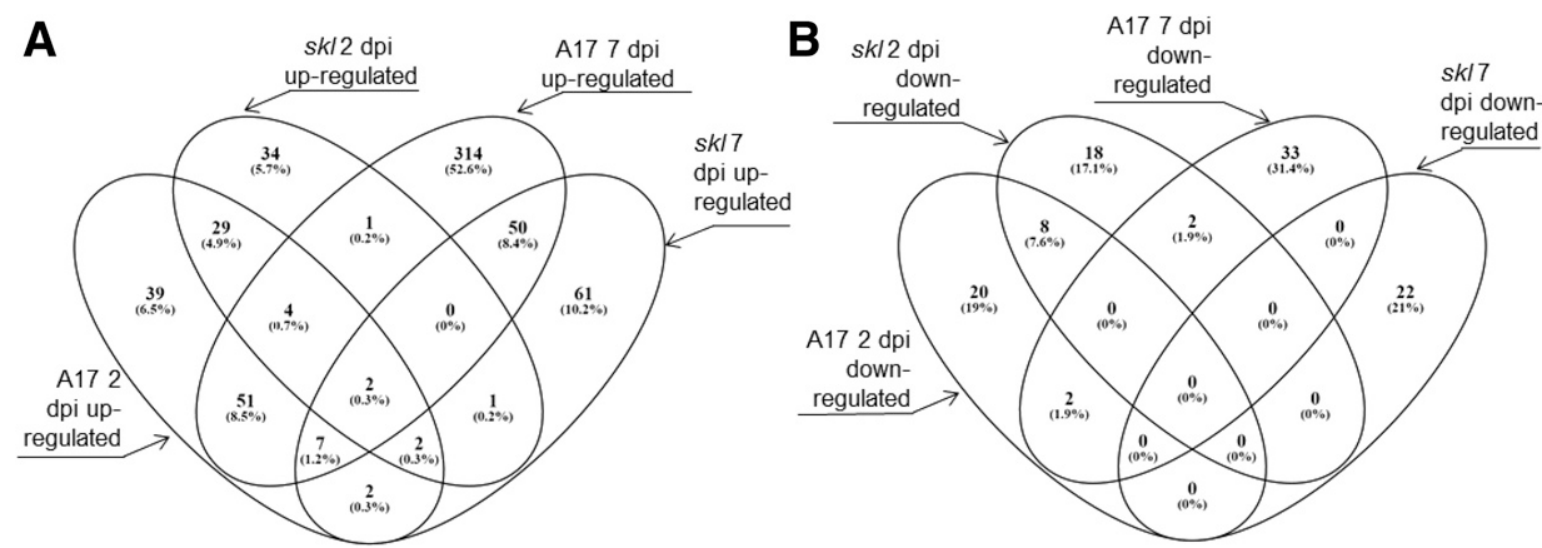

Fig. 3. Gene regulation in A17 and $s k l$ at 2 and 7 days postinoculation (dpi) with Rhizoctonia solani AG8. The total number of genes are indicated in each sector. The percentages of all up- or downregulated genes are presented in parentheses. A, Genes upregulated in inoculated samples compared with mocktreated controls. B, Genes downregulated in inoculated samples compared with mock-treated controls. Only genes significantly differentially expressed (false discovery rate $<0.05$ ) with a $\log 2$-fold change greater than 1 or less than -1 are included. 


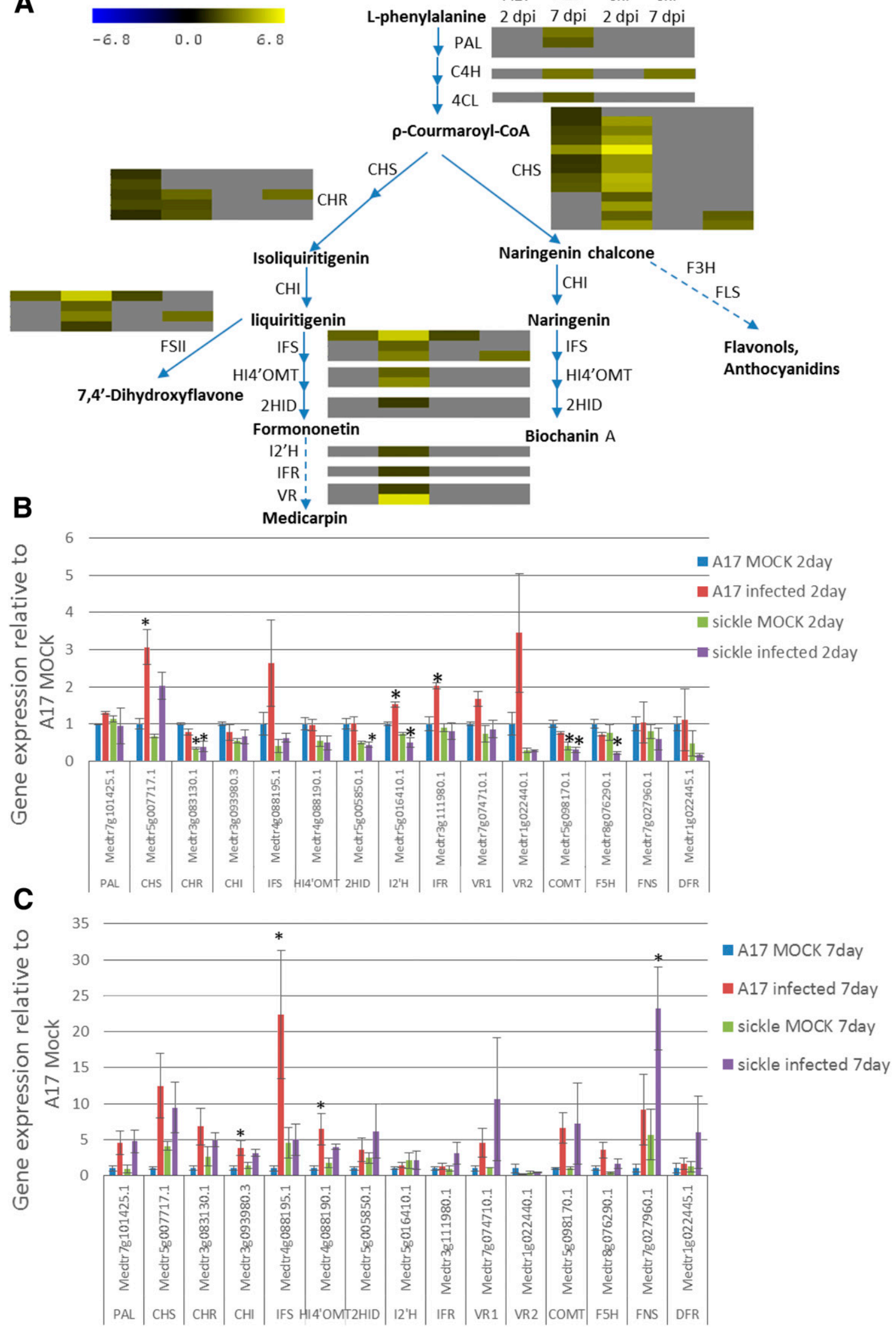

A17 A17 skl skl

$\begin{array}{lll}-6.8 & 0.0 & 6.8\end{array}$

L-phenylalanine $2 \mathrm{dpi} 7 \mathrm{dpi} 2 \mathrm{dpi} 7 \mathrm{dpi}$

Fig. 4. Expression of genes encoding components of the flavonoid pathway. A, A schematic diagram of the flavonoid pathway showing gene expression derived from analysis of RNA-sequencing data represented as heat maps. Only genes with significant differential expression relative to their respective mock samples are shown. The gray color indicates no significant change in expression levels. Heatmap columns show log2-fold induction and, from left to right, are A17 at 2 days postinoculation (dpi), A17 at $7 \mathrm{dpi} ; s k l$ at $2 \mathrm{dpi}$; and $s k l$ at $7 \mathrm{dpi}$. B, Quantitative polymerase chain reaction (qPCR)-based determination of relative gene expression for selected flavonoid pathway genes at 2 and $\mathbf{C}, 7$ dpi. Figures show means and standard error of the means. Asterisks indicate a significant change in expression relative to the A17 mock sample according to two-way analysis of variance with Tukey's post hoc test on qPCR data $(n=3)$. 
A17 and $s k l$ differ in the accumulation of isoflavonoid metabolites in response to $R$. solani inoculation.

To further investigate if transcriptional regulation of genes in flavonoid synthesis pathways correlated with accumulation of the metabolic products of the pathways, we quantified flavonoid metabolites in A17 and skl roots in the absence and presence of $R$. solani at 5 days after infection. As shown in Figure 5, R. solani infection significantly increased the concentrations of liquiritigenin (an isoflavonoid precursor) and the isoflavonoids formononetin, medicarpin, and biochanin $\mathrm{A}$ in A17 roots. In addition, the flavone 7,4'-dihydroxyflavone, also derived from liquiritigenin, was significantly higher in infected A17 roots.

In contrast, roots of the $s k l$ mutant showed a modest increase in formononetin and biochanin A but not in medicarpin or 7,4'dihydroxyflavone (Fig. 5). Instead, skl roots accumulated increased concentrations of the isoflavonoid precursors isoliquiritigenin and naringenin in response to $R$. solani infection. Together with the previous gene expression data, these findings suggest the $s k l$ mutant might be defective in the full deployment of the isoflavonoid pathway in response to $R$. solani infection.
Overexpression of the isoflavonoid pathway

in A17 enhances resistance to $R$. solani.

To investigate the role of isoflavonoid-derived phytoalexins in defense against $R$. solani, the isoflavone synthase-encoding IFS gene, which is both expressed in roots and responsive to $R$. solani inoculation in A17, was overexpressed in transgenic hairy roots of $M$. truncatula A17 that were inoculated with $R$. solani. The transgenic roots containing the IFS overexpression construct (IFS-Ox) showed increased expression of the IFS gene relative to the empty vector control and increased concentrations of the isoflavonoid formononetin (Supplementary Fig. S5). Infection of $M$. truncatula hairy roots with $R$. solani led to a reduction in root growth and continued colonization lead to collapse of the plant. Overexpression of IFS in IFS-Ox roots resulted in a significant increase in root growth after $R$. solani infection compared with the control (Fig. 6A). In addition, IFS-Ox roots demonstrated a significant increase in secondary root emergence downstream of the inoculation point (Fig. 6B), suggesting that IFS overexpression led to enhanced resistance to $R$. solani infection. Collectively, the specific upregulation of the isoflavonoid biosynthesis pathway in A17 as well as the hairy root infection
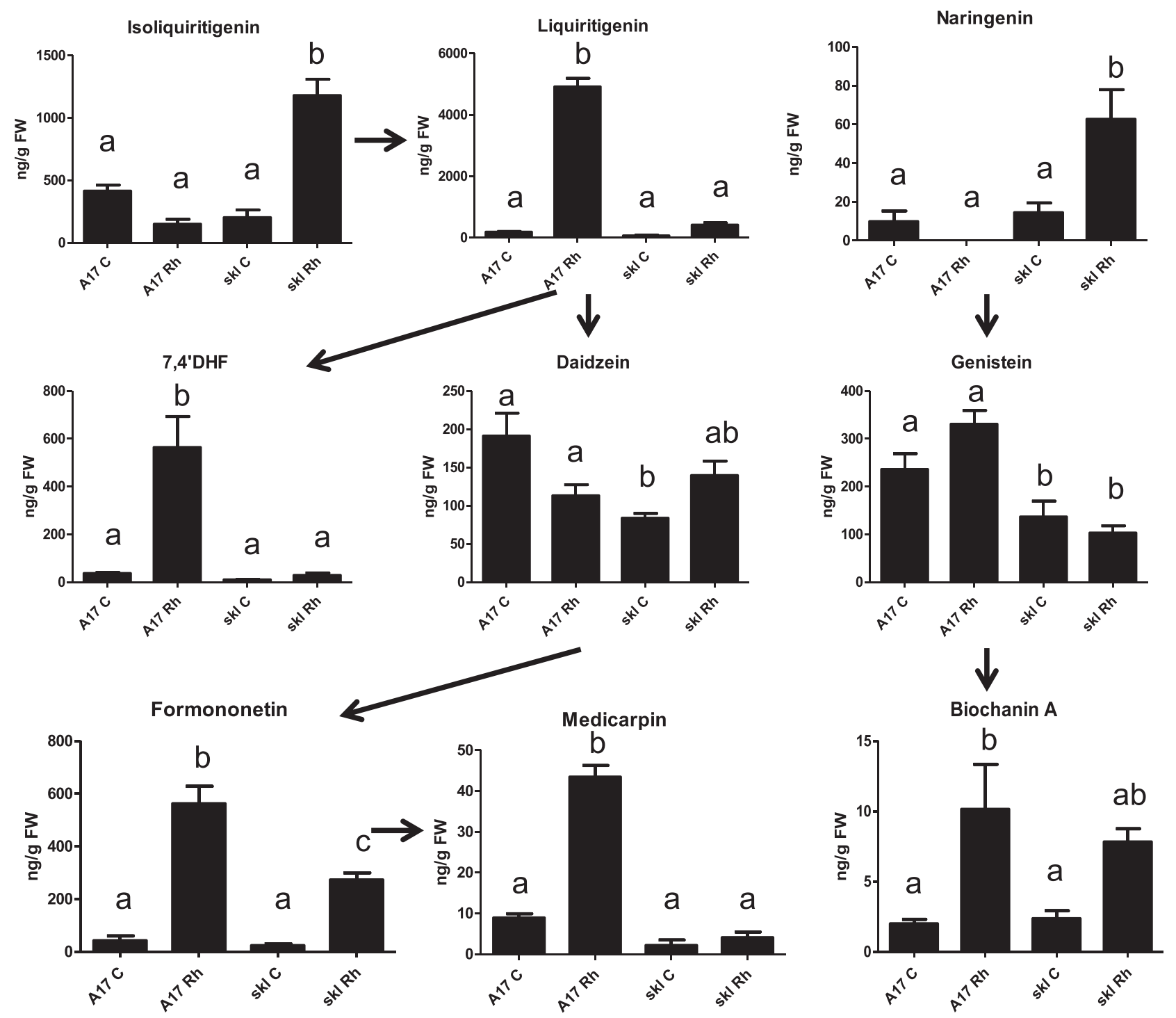

Fig. 5. Accumulation of flavonoids in A17 and skl roots in response to Rhizoctonia solani inoculation. Flavonoid accumulation was quantified in infected and noninfected roots of A17 and $s k l$ seedling roots 5 days after infection compared with authentic standards. The arrows indicate conversions of flavonoid precursors to various end products. Each figure shows means and standard error of the means; bars designated with different letters differ significantly at $P<$ 0.05 , according to analysis of variance with Tukey's post hoc test $(n=3)$. 
results suggest that IFS-derived metabolites may confer increased resistance to $R$. solani infection.

In addition to the overexpression assays, we tested whether supplementation of roots with the isoflavonoid end product medicarpin, which was significantly increased in A17 roots but not in $s k l$ roots in response to $R$. solani, would reduce disease symptoms in response to $R$. solani. For these assays, A17 and $s k l$ seedlings were grown under sterile conditions on Petri dishes containing nutrient agar and were infected with plugs of $R$. solani after treatment with either solvent or $10 \mu \mathrm{M}$ medicarpin solution. Although no significant improvement in plant performance was observed, supplementation of roots with medicarpin resulted in a trend toward reduced root browning (Supplementary Figs. S6 and S7) and a partial increase in lateral root formation and shoot weight at 7 days after $R$. solani infection, in particular in the $s k l$ mutant. Medicarpin addition did not rescue root fresh weight or tap root growth in infected roots of either genotype. Compared with the resistance phenotype observed after IFS overexpression, these results suggest that lack of medicarpin induction in $s k l$ roots in response to $R$. solani might be one of several factors making this mutant more susceptible to infection.

\section{DISCUSSION}

The necrotrophic fungal pathogen $R$. solani causes disease on all of the world's major food crops, including many legumes. Legumes play an important role in agriculture through symbiotic nitrogen fixation and the disease break advantages they offer in crop rotation strategies. To date, no strong, effective resistance to $R$. solani has been observed in any crop species. The interaction of the model legume $M$. truncatula and $R$. solani can be used as a model pathosystem to provide insights into legume defense mechanisms and ultimately to formulate strategies to minimize the impact of $R$. solani disease on legume hosts and, potentially, other crops.

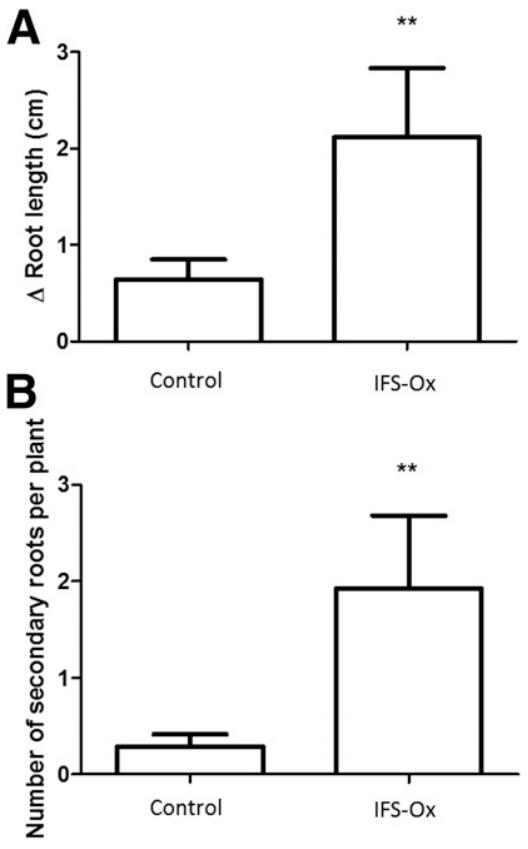

Fig. 6. Root phenotypes of A17 plants with transgenic roots overexpressing isoflavone synthase. A, The change $(\Delta)$ in root growth from the start of inoculation and $\mathbf{B}$, lateral root emergence since infection was quantified after 14 days. Figures show means and standard error of the means; asterisks $(* *)$ indicate significant changes between control (empty vector) and isoflavone synthase-overexpressing roots (IFS-Ox) $(P<0.001$; Student's $t$ test; $n>40)$. An independent repeat experiment showed similar results.
We have previously observed moderate resistance in the M. truncatula reference accession A17 and high susceptibility in the ethylene insensitive and hypernodulating mutant $s k l$ (Anderson et al. 2010; Penmetsa et al. 2008). Comparison of the basal gene expression in A17 and $s k l$ seedlings in the absence of biotic stresses revealed wide-ranging changes in gene expression; however, few GO categories were statistically overrepresented among these genes compared with the genome as a whole. Overall, the large difference in basal gene expression may relate to differences in growth and development observed between these plants and, although they are in similar environments, the ethylene insensitivity in $s k l$ appears to be causing the plants to adapt to the environment differently than does A17.

Larrainzar et al. (2015) used RNA-seq to study gene expression in A17, skl, and the nod factor perception mutants $n f p$ and $l y k 3$ prior to the addition of Sinorhizobium spp. and during a detailed time course thereafter. Of the genes found to be differentially expressed in the current study, $90 \%$ of those that showed downregulation in $s k l$ and $65 \%$ of the genes showing upregulation in $s k l$ showed a similar expression pattern (Larrainzar et al. 2015). Overall, these findings confirm the scale of difference we observed between A17 and skl in the absence of a biotic interaction, despite our study being performed in vermiculite and Larrainzar et al. (2015) using an aeroponic system.

Although A17 showed a substantially larger number of genes responding to $R$. solani infection at 7 days after inoculation, $s k l$ showed a more modest increase at the later time point (Fig. 3). This difference in the 7-day response may relate to the severity of infection occurring in these tissues, with A17 scaling up a defense response and $s k l$ succumbing to an overwhelming infection. One clear difference between the responses of A17 and $s k l$ was in the activation of the ethylene pathway. A17 recruited this pathway early and with greater magnitude by 7 days after infection. This finding is consistent with previous studies on the ethylene insensitivity of $s k l$ and the pathway's early role in defense against $R$. solani in A17 (Anderson and Singh 2011; Anderson et al. 2010; Penmetsa et al. 2008).

Besides the specific induction of the ethylene signaling pathway in A17 following $R$. solani challenge, the upregulation of secondary metabolism pathways was another notable difference between A17 and skl. Further dissection of these secondary metabolism pathways identified genes associated with the production of flavonoids, especially genes leading to the synthesis of the antimicrobial isoflavonoids, were specifically induced in A17 following $R$. solani infection. IFS (EC 1.14.13.136), isoflavone 2 '-hydroxylase (I2'H; EC:1.14.13.89), and isoflavone reductase (EC 1.3.1.45) are isoflavonoidspecific enzymes involved in the biosynthesis of phytoalexins such as medicarpin (López-Meyer and Paiva 2002). These genes and several genes in precursor pathways showed a specific upregulation in A17 following $R$. solani challenge, suggesting specific accumulation of isoflavonoids in an ethylene-dependent manner.

To further examine the isoflavonoids produced by $M$. truncatula under pathogen attack, the levels of several isoflavonoids and isoflavonoid-associated compounds were quantified. In contrast to the $s k l$ mutant, $R$. solani-challenged A17 accumulated higher levels of liquiritigenin (an isoflavonoid precursor) and the isoflavonoids formononetin, medicarpin, and biochanin A. Furthermore, overexpression of IFS in M. truncatula roots led to higher isoflavonoid accumulation and reduced impact of $R$. solani infection relative to empty-vector controls (Fig. 6). Taken together, these findings provide strong support for isoflavonoids, such as medicarpin, to be contributing to $R$. solani resistance in $M$. truncatula. 
Medicarpin has previously been shown to be involved in the defense response in $M$. truncatula against various pathogens. For example the medicarpin biosynthesis pathway was induced following inoculation of M. truncatula with Phoma medicaginis (Kamphuis et al. 2012b) and Jasiński et al. (2009) found medicarpin precursors to accumulate 1 to 3 days post$P$. medicaginis infection. Medicarpin itself was found at higher levels at 5 days postinfection relative to noninfected leaves. Furthermore, Toyoda et al. (2013) found medicarpin accumulation was induced following infection of $M$. truncatula with Mycosphaerella pinodes. In alfalfa, $M$. sativa, inoculation with an avirulent isolate of Colletotrichum trifolii induced the expression of isoflavonoid biosynthesis pathway genes and led to accumulation of the phytoalexins medicarpin and sativan. These plants also showed protection from additional exposure to a virulent fungal race (Saunders and O'Neill 2004).

Although overexpression of IFS in M. truncatula roots led to greater resistance to $R$. solani, this could be through the activity of several products of this pathway, of which medicarpin is one. To investigate if medicarpin by itself could account for the resistance, the purified compound was added to A17 and skl prior to inoculation with $R$. solani. Although a trend for enhanced resistance to $R$. solani could be observed in the medicarpin-treated plants in measures such as root browning and lateral root formation after infection, particularly for $s k l$, no significant differences were observed between control or medicarpin-treated plants after $R$. solani inoculation, suggesting medicarpin alone is insufficient to confer the moderate resistance observed in A17. A combination of isoflavonoids such as medicarpin, biochanin A, and potentially novel isoflavonoids may be required for protection from $R$. solani, as observed in the IFS-overexpressing $M$. truncatula roots. Biochanin A and dihydroderivatives of biochanin A have been shown to have antimicrobial properties against soybean-infecting isolates of $R$. solani (Weidenbörner et al. 1990). The early induction of these isoflavonoid biosynthesis pathways in A17 may slow pathogen infection, thereby providing a greater window of opportunity to defend against the pathogen. It's also possible that the induction of these compounds needs to be localized to the infection site at the correct infection stage to prevent the infection from progressing into the root cortex.

Although enhanced resistance to $R$. solani was obtained from overexpressing IFS in M. truncatula, manipulation of this pathway to achieve greater root-pathogen resistance must balance any impact on beneficial plant-microbe interactions also occurring in this tissue. Guenoune et al. (2001) observed that Rhizophagus irregularis was sensitive to low concentrations of medicarpin and that roots with sufficient phosphorous supply showed accumulation of medicarpin that correlated with the absence of colonization by the beneficial fungus. Therefore suppression of isoflavonoids is required for mycorrhizal colonization (Harrison and Dixon 1994; Volpin et al. 1995). In addition, while isoflavones are important signals for nodulation of soybean by Bradyrhizobium japonicum, isoflavonoids such as medicarpin, coumestrol, and genistein inhibit the regulation of Sinorhizobium meliloti nod genes (Kosslak et al. 1987; Zuanazzi et al. 1998). The important symbiotic interactions occurring in the roots of legumes must be balanced with the requirement for protection from potential challenges from root-infecting fungal pathogens such as $R$. solani, suggesting legumes must employ an efficient system of checks and balances to optimize growth under a wide range of conditions.

The ethylene-mediated defense responses induced in A17 following $R$. solani infection play a significant role in determining the outcome of the plant-pathogen interaction. The genome-wide transcriptome analysis presented herein suggests a major difference between the responses of A17 and $s k l$ lies in the specific recruitment of isoflavonoid biosynthesis pathways. Metabolite analysis revealed higher levels of isoflavonoidrelated compounds only in A17 following pathogen infection and overexpression of the IFS gene demonstrated the importance of isoflavonoids in resistance to $R$. solani. The activation of these defense responses in roots is likely to impact symbiotic interactions occurring in the same tissues and, thus, a tight temporal and spatial regulation of the defense responses in roots tissues is likely to be important. Early and efficient activation of defense pathways is, therefore, a key factor in quantitative resistance responses to root-infecting pathogens and could be a target of crop improvement strategies for legumes.

\section{MATERIALS AND METHODS}

\section{Plant growth and fungal infections.}

M. truncatula lines A17 and skl were treated, grown, and inoculated as previously described (Anderson et al. 2010). $R$. solani AG8 (isolate WAC10335) was prepared according to Lichtenzveig et al. (2006). Briefly, surface-sterilized $M$. truncatula seeds were germinated on moist filter paper in the dark at $4^{\circ} \mathrm{C}$ for 2 days, then for 1 day at $24^{\circ} \mathrm{C}$. Seedlings were added to pots half filled with vermiculite also containing $5 \mathrm{ml}$ of a $2.2-\mathrm{mg} / \mathrm{ml}$ dry weight equivalent suspension of homogenized fungal mycelium. The seedlings were covered with fine vermiculite and were transferred to growth rooms at a constant temperature of $24^{\circ} \mathrm{C}$ with a 16-h light and 8-h dark photoperiod at a light intensity of $200 \mu \mathrm{mol} \mathrm{m} \mathrm{m}^{-2} \mathrm{~s}^{-1}$. Mocktreated plants were treated the same, with the addition of $5 \mathrm{ml}$ of sterile water rather than the $5 \mathrm{ml}$ of $R$. solani suspension. Inoculated plants were harvested at 2 and 7 days after planting into preinoculated pots, with corresponding mock samples taken at the same time. Each sample consisted of a pool of 16 plants. Root and above-ground tissue was collected separately, was immediately frozen in liquid nitrogen, and was stored at $-80^{\circ} \mathrm{C}$, until RNA extraction. The remaining plants were scored for survival and were harvested at 21 days after inoculation to confirm the infection had progressed as previously described (Anderson et al. 2013). Quantification of in-planta fungal DNA was conducted according to Foley et al. (2013). All experiments included three biological replicates; only root tissue was used for gene expression analysis.

\section{RNA extractions and RNA sequencing.}

Frozen root tissue was ground in the presence of liquid nitrogen and total RNA was extracted, using Trizol reagent (Sigma) according to manufacturer's instructions. Library preparation and sequencing used the TruSeq stranded total RNA library kit (Illumina), and the libraries were sequenced on an Illumina HiSeq 2000, to generate between 5.5 and $24 \mathrm{~Gb}$ of clean reads per library.

\section{Data analysis.}

RNA-seq paired-end reads were trimmed for Illumina adapter sequences, with reads trimmed to less than 25 bp discarded, using Cutadapt (Martin 2011) (parameters: format, fastq; overlap, 10; times, 3; minimum length, 25). Trimmed RNA-seq reads were then mapped to $M$. truncatula genome version 4.0 (Tang et al. 2014), using Tophat2 (parameters: b2, very-sensitive; r, 80; mate-std-dev, 40; i, 20; I, 5000; g, 20; report secondary alignments; $\mathrm{m}, 0$; min coverage intron, 20; microexon search; library type, fr-firststrand) (Trapnell et al. 2009, 2012). BAM files generated from Tophat 2 output were sorted using SAMtools version 0.1.19 (Li et al. 2009). Read alignment details are presented in Supplementary Table S3.

Htseq-count (Anders et al. 2015) was used to count aligned reads per gene, and the resulting count matrix was normalized 
and analyzed for differential expression using the EdgeR package (Robinson et al. 2010) in $\mathrm{R}$ version 3.2.2, using a 0.05 FDR cut-off to determine differentially expressed genes. Smear plots were generated using EdgeR (Supplementary Fig. S8).

Genes showing significant differential expression between samples and a log base twofold change greater than 1 were input into Venny (Oliveros 2007), for identification of genes shared or unique to each sample type. Genes showing differential expression between samples were input into AGRIGO singular enrichment analysis (Du et al. 2010), using Fishers exact test with Yekutieli multitest adjustment to identify overrepresented GO terms among the genes. GOslimviewer (McCarthy et al. 2006) and KOBAS version 2.0 (Xie et al. 2011), with default parameters and species set to Medicago truncatula, were used to display all GO terms and KEGG pathways associated with a set of differentially expressed genes. Multiple Expression Viewer version 4.9.0 (Saeed et al. 2003) was used to generate hierarchical clustered heatmap diagrams with optimized gene leaf order, using Pearson correlation with average linkage clustering, and MapMan version 3.5.1R2 (Thimm et al. 2004) was used to observe differential activity of pathways in different samples, using the MT4.0_v1 mapping.

\section{qPCR analysis of gene expression.}

RNA was confirmed to be free from genomic DNA contamination, by PCR with the tubulin primers, prior to production of cDNA. Reverse transcription of RNA was performed as previously described (Gao et al. 2007). qPCR and calculation of relative gene expression was performed as previously described (Anderson et al. 2010, 2016). Tubulin (Medtr4g019110) and actin (Medtr3g095530) were used as internal controls and have previously been assessed as stably expressed genes among various control and pathogen-infected tissues (Anderson et al. 2010; Gao et al. 2007; Kakar et al. 2008; Williams et al. 2016). Data were analyzed by two-way analysis of variance (ANOVA) with post hoc Tukey honest significant difference tests, using the JMP 7.0 software package (SAS Institute). Primers are listed in Supplementary Table S4.

\section{Overexpression of IFS in $\mathbf{M}$. truncatula hairy roots.}

IFS from M. truncatula (AY939826.1/Medtr4g088195.1) was overexpressed under the control of the Cauliflower mosaic virus $35 \mathrm{~S}$ promoter in transgenic hairy roots, using the vector pK7WG2D (Karimi et al. 2002). The full-length sequences of these genes were obtained from the Medicago Gene Index (Dana Farber Cancer Institute). The expression of the various copies of the same gene was searched in the Medicago Gene Expression Atlas (Noble Foundation). The gene copy that was expressed consistently in root samples in multiple experiments was selected for overexpression. The primers 5'-ATGTT GGTGGAACTTGCAGT-3' and 5'-TTAGGAGGAAAGAAGT TTAT $-3^{\prime}$ in the forward and reverse directions were used to amplify the gene. The $a t t B$ sites were added to the primers in the forward and reverse direction for Gateway cloning (Life Technologies). The resulting clone was inserted into the vector pDONR221, was subcloned into the pK7WG2D vector, and was transformed into Agrobacterium rhizogenes ARqua1. The vector was confirmed by sequencing. $M$. truncatula hairy root transformation with the overexpression and empty-vector plasmid were carried out as described previously using the A17 genotype (Boisson-Dernier et al. 2001). The plates containing transformed plantlets were transferred to a growth room at $25^{\circ} \mathrm{C}$, with a light intensity of $150 \mu \mathrm{mol} \cdot \mathrm{m}^{-2} \cdot \mathrm{s}^{-1}$ for $16 \mathrm{~h}$ per day. Hairy roots were screened for green fluorescent protein fluorescence, using a Leica M205 FA stereomicroscope with an
ET Blue LP filter system (maximum excitation at $470 \mathrm{~nm}$ with a 515-nm long pass filter), which was used as a visual marker for transformation.

Expression levels of IFS in the transgenic roots was quantified by real-time PCR using the following primers: F, 5'-GA TCGACGGGTATGTGGTTC3-' and R, 5'-CTGCTTCACCT TCACCAACA-3'. The root samples were snap-frozen in liquid nitrogen and were ground to a fine powder using a mortar and pestle. RNA was extracted using the Spectrum plant total RNA kit (Sigma) as per manufacturer's instruction followed by oncolumn DNase digestion (Sigma). The extracted RNA was quantified on a NanoDrop ND1000 UV/Vis spectrophotometer (Thermo Fisher Scientific). The first-strand cDNA was synthesized from an equal amount of RNA from all the samples, using the Superscript III first strand cDNA synthesis kit (Life Technologies). The gene fragments were amplified using ABI Power SYBR green PCR master mix kit (Applied Biosystems) on the ABI 7900HT sequence detection system (Applied Biosystems). Thermocycling conditions followed the manufacturers' recommendation for the ABI Power SYBR green PCR master mix kit (Applied Biosystems). The M. truncatula GAPDH gene (MTR_3g085850) (Kakar et al. 2008) was used as a reference control. Primers used for the reference gene were F, 5'-TGCCTACCGTCGATGTTTCAGT-3' and R 5'-TTGCC CTCTGATTCCTCCTTG- ${ }^{\prime}$. Flavonoid concentrations in the transgenic roots was quantified as described below.

\section{Rhizoctonia infection assessment of plants after treatment with medicarpin.}

$M$. truncatula seeds were scarified by immersion in concentrated sulphuric acid for $3 \mathrm{~min}$, were rinsed five times in water, and were sterilized in $6 \%$ (wt/vol) sodium hypochlorite for $10 \mathrm{~min}$ with agitation, followed by five rinses in sterile water. The seeds were imbibed by immersion in water for $1 \mathrm{~h}$, were plated on water agar plates, were placed at $4^{\circ} \mathrm{C}$ for $24 \mathrm{~h}$, and were then transferred to $20^{\circ} \mathrm{C}$ for $12 \mathrm{~h}$, to allow seed germination. Seedlings of $1 \mathrm{~cm}$ in length were placed on Fåhraeus agar media (eight seedlings per $15-\mathrm{cm}$ diameter plate constituted one biological replicate) and were grown in an incubator for 2 days at $25^{\circ} \mathrm{C}\left(16-\mathrm{h}\right.$ day length, $150 \mu \mathrm{mol} \cdot \mathrm{m}^{-2} \cdot \mathrm{s}^{-1}$ light intensity). Seedlings were then treated with $100 \mu \mathrm{l}$, each, of $10 \mu \mathrm{M}$ medicarpin (Apin Chemicals) by pipetting the filtersterilized solution along each seedling root $(0.5 \%$ dimethyl sulfoxide [vol/vol] was used as a solvent control). The solution was allowed to soak into the agar adjoining the roots for $2 \mathrm{~h}$. A fresh plug of $R$. solani culture growing on potato dextrose agar (PDA) was then placed at the root tip of each seedling and the position of the root tip was marked. Sterile PDA plugs were used as controls. Root length was measured, lateral root numbers were counted, root and shoot fresh weights were determined with $0.001 \mathrm{~g}$ accuracy, and the browning index was determined. Data were analyzed using either ANOVA with a Tukey's post hoc test or Kruskal-Wallis test with Dunn's multiple comparison test for the browning index.

\section{Quantification of flavonoids.}

Roots were excised and at least eight roots were pooled per sample. Three biological replicates were prepared for each genotype and treatment. Roots were weighed to $100 \mathrm{mg}$ each, were ground in liquid nitrogen using a mortar and pestle, and were then freeze-dried. Umbelliferone (Sigma Chemicals) was added as an internal standard ( $25 \mu \mathrm{l}$ of $1 \mathrm{ppm})$ and $1 \mathrm{ml}$ of $80 \%$ high-pressure liquid chromatography-grade methanol was added to each freeze-dried sample. The mixture was sonicated for $30 \mathrm{~min}$ at $4^{\circ} \mathrm{C}$ and was centrifuged for $10 \mathrm{~min}$ at $500 \times \mathrm{g}$. The supernatant was then removed and was dried in a vacuum centrifuge. The pellet was re-extracted a second time. The dried 
supernatant was dissolved in $50 \mu \mathrm{l}$ of $80 \%$ (vol/vol) methanol and was filtered through a Nanosep MF GHP $0.45-\mu \mathrm{m}$ filter (Pall Life Sciences). The samples were then analyzed using tandem mass spectrometry, following the method described by (Ng et al. 2015). Data analysis was performed using the Agilent Technologies MassHunter software, followed by ANOVA with Tukey's post hoc test.

\section{Accession numbers.}

Sequence data from this work can be found in the Gene Expression Omnibus database under accession number GSE94260 and the ArrayExpress database under accession number E-MTAB-5473.

\section{ACKNOWLEDGMENTS}

The authors thank N. Pain and H. Casarotto for technical assistance. The work was supported by the Commonwealth Scientific and Industrial Research Organisation, The Grains Research and Development Corporation, The Australian National University, an Australian Research Council scholarship to S. Hassan and a Chinese Scholarship Council scholarship to Y. Liu.

\section{LITERATURE CITED}

Anders, S., Pyl, P. T., and Huber, W. 2015. HTSeq-A Python framework to work with high-throughput sequencing data. Bioinformatics 31:166-169. Anderson, J. P., Hane, J. K., Stoll, T., Pain, N., Hastie, M. L., Kaur, P., Hoogland, C., Gorman, J. J., and Singh, K. B. 2016. Proteomic analysis of Rhizoctonia solani identifies infection-specific, redox associated proteins and insight into adaptation to different plant hosts. Mol. Cell. Proteomics 15:1188-1203.

Anderson, J. P., Lichtenzveig, J., Gleason, C., Oliver, R. P., and Singh, K. B. 2010. The B-3 ethylene response factor MtERF1-1 mediates resistance to a subset of root pathogens in Medicago truncatula without adversely affecting symbiosis with rhizobia. Plant Physiol. 154:861-873.

Anderson, J. P., Lichtenzveig, J., Oliver, R. P., and Singh, K. B. 2013. Medicago truncatula as a model host for studying legume infecting Rhizoctonia solani and identification of a locus affecting resistance to root canker. Plant Pathol. 62:908-921.

Anderson, J. P., and Singh, K. B. 2011. Interactions of Arabidopsis and $M$. truncatula with the same pathogens differ in dependence on ethylene and ethylene response factors. Plant Signal. Behav. 6:551-552.

Bent, A. F., Innes, R. W., Ecker, J. R., and Staskawicz, B. J. 1992. Disease development in ethylene-insensitive Arabidopsis thaliana infected with virulent and avirulent Pseudomonas and Xanthomonas pathogens. Mol. Plant-Microbe Interact 5:372-378.

Boisson-Dernier, A., Chabaud, M., Garcia, F., Bécard, G., Rosenberg, C., and Barker, D. G. 2001. Agrobacterium rhizogenes-transformed roots of Medicago truncatula for the study of nitrogen-fixing and endomycorrhizal symbiotic associations. Mol. Plant-Microbe Interact 14:695-700.

Bonhomme, M., André, O., Badis, Y., Ronfort, J., Burgarella, C., Chantret, N., Prosperi, J.-M., Briskine, R., Mudge, J., Debéllé, F., Navier, H., Miteul, H., Hajri, A., Baranger, A., Tiffin, P., Dumas, B., Pilet-Nayel, M.-L., Young, N. D., and Jacquet, C. 2014. High-density genome-wide association mapping implicates an F-box encoding gene in Medicago truncatula resistance to Aphanomyces euteiches. New Phytol. 201:1328-1342.

Bravo, A., York, T., Pumplin, N., Mueller, L. A., and Harrison, M. J. 2016 Genes conserved for arbuscular mycorrhizal symbiosis identified through phylogenomics. Nat Plants 2:15208.

Cheng, X., Wang, M., Lee, H. K., Tadege, M., Ratet, P., Udvardi, M., Mysore, K. S., and Wen, J. 2014. An efficient reverse genetics platform in the model legume Medicago truncatula. New Phytol. 201:1065-1076.

Djébali, N., Aribi, S., Taamalli, W., Arraouadi, S., Aouani, M. E., and Badri, M. 2013. Natural variation of Medicago truncatula resistance to Aphanomyces euteiches. Eur. J. Plant Pathol. 135:831-843.

Du, Z., Zhou, X., Ling, Y., Zhang, Z., and Su, Z. 2010. agriGO: A GO analysis toolkit for the agricultural community. Nucleic Acids Res. 38:W64-W70.

Foley, R. C., Gleason, C. A., Anderson, J. P., Hamann, T., and Singh, K. B. 2013. Genetic and genomic analysis of Rhizoctonia solani interactions with Arabidopsis; evidence of resistance mediated through NADPH oxidases. PLoS One 8:e56814.

Foster-Hartnett, D., Danesh, D., Peñuela, S., Sharopova, N., Endre, G., Vandenbosch, K. A., Young, N. D., and Samac, D. A. 2007. Molecular and cytological responses of Medicago truncatula to Erysiphe pisi. Mol. Plant Pathol. 8:307-319.
Fujimoto, S. Y., Ohta, M., Usui, A., Shinshi, H., and Ohme-Takagi, M. 2000. Arabidopsis ethylene-responsive element binding factors act as transcriptional activators or repressors of GCC box-mediated gene expression. Plant Cell 12:393-404.

Gao, L.-L., Anderson, J. P., Klingler, J. P., Nair, R. M., Edwards, O. R., and Singh, K. B. 2007. Involvement of the octadecanoid pathway in bluegreen aphid resistance in Medicago truncatula. Mol. Plant-Microbe Interact 20:82-93.

Gao, L.-L., Klingler, J. P., Anderson, J. P., Edwards, O. R., and Singh, K. B. 2008. Characterization of pea aphid resistance in Medicago truncatula. Plant Physiol. 146:996-1009.

Graham, P. H., and Vance, C. P. 2003. Legumes: Importance and constraints to greater use. Plant Physiol. 131:872-877.

Guenoune, D., Galili, S., Phillips, D. A., Volpin, H., Chet, I., Okon, Y., and Kapulnik, Y. 2001. The defense response elicited by the pathogen Rhizoctonia solani is suppressed by colonization of the AM-fungus Glomus intraradices. Plant Sci. 160:925-932.

Hane, J. K., Anderson, J. P., Williams, A. H., Sperschneider, J., and Singh, K. B. 2014. Genome sequencing and comparative genomics of the broad host-range pathogen Rhizoctonia solani AG8. PLoS Genet. 10: e 1004281 .

Harrison, M. J., and Dixon, R. A. 1994. Spatial patterns of expression of flavonoid/isoflavonoid pathway genes during interactions between roots of Medicago truncatula and the mycorrhizal fungus Glomus versiforme. Plant J. 6:9-20.

Hoffmann, B., Trinh, T. H., Leung, J., Kondorosi, A., and Kondorosi, E. 1997. A new Medicago truncatula line with superior in vitro regeneration, transformation, and symbiotic properties isolated through cell culture selection. Mol. Plant-Microbe Interact 10:307-315.

Jasiński, M., Kachlicki, P., Rodziewicz, P., Figlerowicz, M., and Stobiecki, M. 2009. Changes in the profile of flavonoid accumulation in Medicago truncatula leaves during infection with fungal pathogen Phoma medicaginis. Plant Physiol. Biochem. 47:847-853.

Jones, K. M., Kobayashi, H., Davies, B. W., Taga, M. E., and Walker, G. C. 2007. How rhizobial symbionts invade plants: The SinorhizobiumMedicago model. Nat. Rev. Microbiol. 5:619-633.

Kakar, K., Wandrey, M., Czechowski, T., Gaertner, T., Scheible, W. R., Stitt, M., Torres-Jerez, I., Xiao, Y., Redman, J. C., Wu, H. C., Cheung, F., Town, C. D., and Udvardi, M. K. 2008. A community resource for highthroughput quantitative RT-PCR analysis of transcription factor gene expression in Medicago truncatula. Plant Methods 4:18.

Kamphuis, L. G., Gao, L. L., and Singh, K. B. 2012a. Identification and characterization of resistance to cowpea aphid (Aphis craccivora Koch) in Medicago truncatula. BMC Plant Biol. 12:101.

Kamphuis, L. G., Williams, A. H., Küster, H., Trengove, R. D., Singh, K. B., Oliver, R. P., and Ellwood, S. R. 2012b. Phoma medicaginis stimulates the induction of the octadecanoid and phenylpropanoid pathways in Medicago truncatula. Mol. Plant Pathol. 13:593-603.

Kamphuis, L. G., Zulak, K., Gao, L. L., Anderson, J., and Singh, K. B. 2013. Plant-aphid interactions with a focus on legumes. Funct. Plant Biol. 40:1271-1284.

Karimi, M., Inzé, D., and Depicker, A. 2002. GATEWAY vectors for Agrobacterium-mediated plant transformation. Trends Plant Sci. 7: 193-195.

Klingler, J. P., Edwards, O. R., and Singh, K. B. 2007. Independent action and contrasting phenotypes of resistance genes against spotted alfalfa aphid and bluegreen aphid in Medicago truncatula. New Phytol. 173: 630-640.

Kosslak, R. M., Bookland, R., Barkei, J., Paaren, H. E., and Appelbaum, E. R. 1987. Induction of Bradyrhizobium japonicum common nod genes by isoflavones isolated from Glycine max. Proc. Natl. Acad. Sci. U.S.A. 84:7428-7432.

Larrainzar, E., Riely, B. K., Kim, S. C., Carrasquilla-Garcia, N., Yu, H. J., Hwang, H. J., Oh, M., Kim, G. B., Surendrarao, A. K., Chasman, D., Siahpirani, A. F., Penmetsa, R. V., Lee, G. S., Kim, N., Roy, S., Mun, J. H., and Cook, D. R. 2015. Deep sequencing of the Medicago truncatula root transcriptome reveals a massive and early interaction between nodulation factor and ethylene signals. Plant Physiol. 169:233-265.

Leitner, M., Boland, W., and Mithöfer, A. 2005. Direct and indirect defences induced by piercing-sucking and chewing herbivores in Medicago truncatula. New Phytol. 167:597-606.

Li, H., Handsaker, B., Wysoker, A., Fennell, T., Ruan, J., Homer, N., Marth, G., Abecasis, G., and Durbin, R.; 1000 Genome Project Data Processing Subgroup. 2009. The Sequence Alignment/Map format and SAMtools. Bioinformatics 25:2078-2079.

Lichtenzveig, J., Anderson, J. P., Thomas, G., Oliver, R., and Singh, K. B. 2006. Inoculation and growth with soil borne pathogenic fungi. The Samuel Roberts Noble Foundation, Ardmore, OK, U.S.A. 
López-Meyer, M., and Paiva, N. L. 2002. Immunolocalization of vestitone reductase and isoflavone reductase, two enzymes involved in the biosynthesis of the phytoalexin medicarpin. Physiol. Mol. Plant Pathol. 61:15-30.

Martin, M. 2011. Cutadapt removes adapter sequences from highthroughput sequencing reads. EMBnet.journal 17:10-12.

McCarthy, F. M., Wang, N., Magee, G. B., Nanduri, B., Lawrence, M. L., Camon, E. B., Barrell, D. G., Hill, D. P., Dolan, M. E., Williams, W. P., Luthe, D. S., Bridges, S. M., and Burgess, S. C. 2006. AgBase: A functional genomics resource for agriculture. BMC Genomics 7:229.

Ng, J. L. P., Hassan, S., Truong, T. T., Hocart, C. H., Laffont, C., Frugier, F., and Mathesius, U. 2015. Flavonoids and auxin transport inhibitors rescue symbiotic nodulation in the Medicago truncatula cytokinin perception mutant cre1. Plant Cell 27:2210-2226.

Oliveros, J. C. 2007. VENNY. An interactive tool for comparing lists with Venn diagrams. BioinfoGP, Spanish National Biotechnology Center, Madrid. http://bioinfogp.cnb.csic.es/tools/venny/index.html.

Penmetsa, R. V., Uribe, P., Anderson, J., Lichtenzveig, J., Gish, J.-C., Nam, Y. W., Engstrom, E., Xu, K., Sckisel, G., Pereira, M., Baek, J. M., LópezMeyer, M., Long, S. R., Harrison, M. J., Singh, K. B., Kiss, G. B., and Cook, D. R. 2008. The Medicago truncatula ortholog of Arabidopsis EIN2, sickle, is a negative regulator of symbiotic and pathogenic microbial associations. Plant J. 55:580-595.

Robinson, M. D., McCarthy, D. J., and Smyth, G. K. 2010. edgeR: A Bioconductor package for differential expression analysis of digital gene expression data. Bioinformatics 26:139-140.

Saeed, A. I., Sharov, V., White, J., Li, J., Liang, W., Bhagabati, N., Braisted, J., Klapa, M., Currier, T., Thiagarajan, M., Sturn, A., Snuffin, M., Rezantsev, A., Popov, D., Ryltsov, A., Kostukovich, E., Borisovsky, I., Liu, Z., Vinsavich, A., Trush, V., and Quackenbush, J. 2003. TM4: A free, open-source system for microarray data management and analysis. Biotechniques 34:374-378.

Saunders, J. A., and O'Neill, N. R. 2004. The characterization of defense responses to fungal infection in alfalfa. BioControl 49:715-728.

Stewart, S. A., Hodge, S., Ismail, N., Mansfield, J. W., Feys, B. J., Prospéri, J. M., Huguet, T., Ben, C., Gentzbittel, L., and Powell, G. 2009. The $R A P 1$ gene confers effective, race-specific resistance to the pea aphid in Medicago truncatula independent of the hypersensitive reaction. Mol. Plant-Microbe Interact 22:1645-1655.

Tang, H., Krishnakumar, V., Bidwell, S., Rosen, B., Chan, A., Zhou, S., Gentzbittel, L., Childs, K. L., Yandell, M., Gundlach, H., Mayer, K. F. X., Schwartz, D. C., and Town, C. D. 2014. An improved genome release (version Mt4.0) for the model legume Medicago truncatula. BMC Genomics 15:312.

Thatcher, L. F., Williams, A. H., Garg, G., Buck, S. G., and Singh, K. B. 2016. Transcriptome analysis of the fungal pathogen Fusarium oxysporum f. sp. medicaginis during colonisation of resistant and susceptible Medicago truncatula hosts identifies differential pathogenicity profiles and novel candidate effectors. BMC Genomics 17:860.

Thimm, O., Bläsing, O., Gibon, Y., Nagel, A., Meyer, S., Krüger, P., Selbig, J., Müller, L. A., Rhee, S. Y., and Stitt, M. 2004. MAPMAN: A userdriven tool to display genomics data sets onto diagrams of metabolic pathways and other biological processes. Plant J. 37:914-939.

Tivoli, B., Baranger, A., Avila, C. M., Banniza, S., Barbetti, M., Chen, W. D., Davidson, J., Lindeck, K., Kharrat, M., Rubiales, D., Sadiki, M., Sillero, J. C., Sweetingham, M., and Muehlbauer, F. J. 2006. Screening techniques and sources of resistance to foliar diseases caused by major necrotrophic fungi in grain legumes. Euphytica 147:223-253.

Toyoda, K., Ikeda, S., Morikawa, J.-i., Hirose, M., Maeda, A., Suzuki, T., Inagaki, Y., Ichinose, Y., and Shiraishi, T. 2013. The Medicago truncatulaMycosphaerella pinodes interaction: A new pathosystem for dissecting fungal-suppressor-mediated disease susceptibility in plants. J. Gen. Plant Pathol. 79:1-11.
Trapnell, C., Pachter, L., and Salzberg, S. L. 2009. TopHat: Discovering splice junctions with RNA-Seq. Bioinformatics 25:1105-1111.

Trapnell, C., Roberts, A., Goff, L., Pertea, G., Kim, D., Kelley, D. R., Pimentel, H., Salzberg, S. L., Rinn, J. L., and Pachter, L. 2012. Differential gene and transcript expression analysis of RNA-seq experiments with TopHat and Cufflinks. Nat. Protoc. 7:562-578.

Vailleau, F., Sartorel, E., Jardinaud, M. F., Chardon, F., Genin, S., Huguet, T., Gentzbittel, L., and Petitprez, M. 2007. Characterization of the interaction between the bacterial wilt pathogen Ralstonia solanacearum and the model legume plant Medicago truncatula. Mol. Plant-Microbe Interact 20:159-167.

Volpin, H., Phillips, D. A., Okon, Y., and Kapulnik, Y. 1995. Suppression of an isoflavonoid phytoalexin defense response in mycorrhizal alfalfa roots. Plant Physiol. 108:1449-1454.

Weidenbörner, M., Hindorf, H., Jha, H. C., Tsotsonos, P., and Egge, H. 1990. Antifungal activity of isoflavonoids in different reduced stages on Rhizoctonia solani and Sclerotium rolfsii. Phytochemistry 29:801-803.

Williams, A. H., Sharma, M., Thatcher, L. F., Azam, S., Hane, J. K., Sperschneider, J., Kidd, B. N., Anderson, J. P., Ghosh, R., Garg, G., Lichtenzveig, J., Kistler, H. C., Shea, T., Young, S., Buck, S.-A.G., Kamphuis, L. G., Saxena, R., Pande, S., Ma, L.-J., Varshney, R. K., and Singh, K. B. 2016. Comparative genomics and prediction of conditionally dispensable sequences in legume-infecting Fusarium oxysporum formae speciales facilitates identification of candidate effectors. BMC Genomics 17:191.

Wrather, J. A., Anderson, T. R., Arsyad, D. M., Gai, J., Ploper, L. D., PortaPuglia, A., Ram, H. H., and Yorinori, J. T. 1997. Soybean disease loss estimates for the top 10 soybean producing countries in 1994. Plant Dis. $81: 107-110$.

Xie, C., Mao, X., Huang, J., Ding, Y., Wu, J., Dong, S., Kong, L., Gao, G., Li, C. Y., and Wei, L. 2011. KOBAS 2.0: A web server for annotation and identification of enriched pathways and diseases. Nucleic Acids Res. 39: W316-W322.

Young, N. D., Debellé, F., Oldroyd, G. E. D., Geurts, R., Cannon, S. B., Udvardi, M. K., Benedito, V. A., Mayer, K. F. X., Gouzy, J., Schoof, H., Van de Peer, Y., Proost, S., Cook, D. R., Meyers, B. C., Spannagl, M., Cheung, F., De Mita, S., Krishnakumar, V., Gundlach, H., Zhou, S., Mudge, J., Bharti, A. K., Murray, J. D., Naoumkina, M. A., Rosen, B., Silverstein, K. A. T., Tang, H., Rombauts, S., Zhao, P. X., Zhou, P., Barbe, V., Bardou, P., Bechner, M., Bellec, A., Berger, A., Bergès, H., Bidwell, S., Bisseling, T., Choisne, N., Couloux, A., Denny, R. Deshpande, S., Dai, X., Doyle, J. J., Dudez, A.-M., Farmer, A. D., Fouteau, S., Franken, C., Gibelin, C., Gish, J., Goldstein, S., González, A. J., Green, P. J., Hallab, A., Hartog, M., Hua, A., Humphray, S. J., Jeong, D.-H., Jing, Y., Jöcker, A., Kenton, S. M., Kim, D.-J., Klee, K., Lai, H., Lang, C., Lin, S., Macmil, S. L., Magdelenat, G., Matthews, L., McCorrison, J., Monaghan, E. L., Mun, J.-H., Najar, F. Z., Nicholson, C., Noirot, C., O’Bleness, M., Paule, C. R., Poulain, J., Prion, F., Qin, B., Qu, C., Retzel, E. F., Riddle, C., Sallet, E., Samain, S., Samson, N., Sanders, I., Saurat, O., Scarpelli, C., Schiex, T., Segurens, B., Severin, A. J., Sherrier, D. J., Shi, R., Sims, S., Singer, S. R., Sinharoy, S., Sterck, L., Viollet, A., Wang, B. B., Wang, K., Wang, M., Wang, X., Warfsmann, J., Weissenbach, J., White, D. D., White, J. D., Wiley, G. B., Wincker, P., Xing, Y., Yang, L., Yao, Z., Ying, F., Zhai, J., Zhou, L., Zuber, A., Dénarié, J., Dixon, R. A., May, G. D., Schwartz, D. C., Rogers, J., Quétier, F., Town, C. D., and Roe, B. A. 2011. The Medicago genome provides insight into the evolution of rhizobial symbioses. Nature 480:520-524.

Zuanazzi, J. A. S., Clergeot, P. H., Quirion, J. C., Husson, H. P., Kondorosi, A., and Ratet, P. 1998. Production of Sinorhizobium meliloti nod gene activator and repressor flavonoids from Medicago sativa roots. Mol Plant-Microbe Interact 11:784-794. 